\title{
AUDIÇÃO, ZUMBIDO E QUALIDADE DE VIDA: UM ESTUDO PILOTO
}

\author{
Hearing, Tinnitus and Life Quality: a pilot study \\ Carolina Campos Esteves (1), Florence Nunes Brandão (2), \\ Carlos Gustavo Alves Siqueira ${ }^{(3)}$, Sirley Alves da Silva Carvalho ${ }^{(4)}$
}

\begin{abstract}
RESUMO
Objetivos: analisar a influência do zumbido na qualidade de vida dos pacientes; verificar a relação entre grau de incômodo do zumbido e presença de perda auditiva concomitante. Método: estudo observacional transversal com amostra de conveniência. Foram aplicados anamnese e questionário com enfoque das características clínicas do zumbido em 22 pacientes. Para análise dos dados, foi aplicado o Teste exato de Fisher, com nível de significância de 5\% $(p<0,05)$. Resultados: dos 22 pacientes, 7 ( 31,8\%) apresentaram audição normal e 15 (68,2\%) apresentaram algum tipo de perda auditiva. Dos pacientes possuidores de perda auditiva, 12 apresentaram perda auditiva neurossensorial em pelo menos uma das orelhas e 3 perda mista uni ou bilateral. Quanto ao grau de incômodo, utilizando a escala análogo-visual, $17(77,27 \%)$ dos pacientes relataram incômodo intenso, ao passo que $5(22,72 \%)$ consideraram grau reduzido de incômodo gerado por esse sintoma. Foi evidenciado que o zumbido impacta mais as emoções e o sono dos pacientes, do que a concentração e realização de atividades sociais. Conclusões: dos 22 pacientes com zumbido, encaminhados para realização do PEATE, $70 \%$ apresentam zumbido e perda auditiva associada. No entanto, não houve relação entre a presença de zumbido e presença de perda. Não houve ainda relação entre o grau de incômodo e presença de perda.
\end{abstract}

DESCRITORES: Zumbido; Perda Auditiva; Qualidade de Vida; Audiologia

\section{INTRODUÇÃO}

As definições mais recentes de zumbido, encontradas na literatura nacional e internacional, descrevem-no como uma ilusão auditiva, uma sensação sonora endógena, não relacionada a

(1) Fonoaudióloga graduada pela Universidade Federal de Minas Gerais - UFMG, Belo Horizonte, MG, Brasil.

(2) Fonoaudióloga; Especialista em Audiologia Clínica pelo CEFAC - MG.

(3) Fonoaudiólogo graduado pela Universidade Estadual de Ciências da Saúde de Alagoas - UNCISAL; Especialização em Audiologia pela Faculdade de Estudos Administrativos e Faculdade de Estudos Superiores - FEAD, MG.

(4) Fonoaudióloga; Professora Adjunto do Curso de Fonoaudiologia da Universidade Federal de Minas Gerais UFMG; Doutora em Biofísica Sensorial pela Universidade d'Avergne - França.

Estudo apresentado como Trabalho de Conclusão de Curso de Graduação em Fonoaudiologia na Universidade Federal de Minas Gerais - UFMG.

Conflito de interesses: inexistente nenhuma fonte externa de estimulação, sendo provavelmente um sintoma recorrente de outra patologia não auditiva, como alterações metabólicas, que afetam a saúde e o bem-estar do paciente ${ }^{1-3}$. Não se têm dados estatísticos concretos quanto à incidência no Brasil, entretanto acredita-se que mais de 28 milhões de brasileiros apresentam zumbido em alguma etapa de sua vida ${ }^{3,4}$.

Gerado pela porção neurossensorial do sistema auditivo e acometendo aproximadamente um terço da população adulta, o zumbido tem vasto leque de etiologias, entre as quais: otológica, cardiovascular, metabólica, neurológica, farmacológica, odontogênica ou psicogênica. Com o objetivo de diagnosticar a causa do zumbido, os pacientes são encaminhados para avaliação audiológica verificando-se assim a integridade de toda a via auditiva ${ }^{3-6}$.

O modelo neurofisiológico proposto por Jastreboff na década de 90 sugere que o zumbido seria o resultado da interação dinâmica entre os centros auditivos e os não-auditivos do Sistema Nervoso Central, incluindo o Sistema Límbico e o Sistema 
Nervoso Autônomo. A avaliação permanente de um possível estímulo nervoso, pelos Sistemas Nervoso Central e Límbico seria o responsável pelo incômodo induzido pelo zumbido ${ }^{7,8}$. Infere-se que os fatores que geram o seu aparecimento não sejam os mesmos que determinam a sua persistência ou incômodo ${ }^{7,8}$. Este último ocorreria quando a associação entre a percepção do zumbido é aliada a algo negativo, por exemplo: coincidência aleatória da percepção de zumbido e um sentimento de desconforto ou tensão ${ }^{7}$. Pessoas com tal sintoma, frequentemente atribuem ao zumbido muitos de seus problemas, tais como alterações na saúde, depressão e dificuldade de percepção da fala. Tais efeitos podem comprometer de tal maneira a qualidade de vida das pessoas que, em casos extremos, levam ao suicídio ${ }^{3,5,9-11}$.

Muitas pesquisas já foram realizadas a fim de discutir o impacto do zumbido na qualidade de vida ${ }^{3,5,8,9-15}$. Foi demonstrando que $20 \%$ dos pacientes que possuem essa sintomatologia apresentam grau de incômodo moderado a severo, com repercussão impactante e, até mesmo, incapacitante na qualidade de vida, com interferências no sono e nas atividades sociais, além de provocar distúrbios emocionais ${ }^{3,10,16}$. Algumas queixas demonstram que o incômodo do zumbido chega a afetar funções mentais normais, tais como o raciocínio, memória e concentração. Essas alterações trazem prejuízos nas atividades de trabalho e de lazer, no repouso, na comunicação, no ambiente social e doméstico com repercussão na esfera psíquica, deixando as pessoas irritadiças, ansiosas, angustiadas, deprimidas e insones. Fatores, como perda auditiva, alterações concomitantes da orelha média, alterações no sistema vestibular, dores de cabeça, bem como infecções das vias aéreas superiores mostraram papel significante na determinação do grau de incômodo ${ }^{3,9,10}$. Agravantes, como fadiga física e mental, ansiedade, estresse e depressão levam a uma piora no incômodo percebido pelo paciente ${ }^{3,11,12}$.

Ao analisar variáveis, como gênero, idade e presença de perda auditiva tem-se grande variação quanto ao incômodo gerado. Sabe-se que a incidência de zumbido e perda auditiva aumenta com $\mathrm{o}$ avançar da idade ${ }^{2,3,8,11,16}$. A avaliação das consequências do zumbido, por meio de questionários, foi incluída na rotina clínica com o objetivo de quantificar os déficits psicoemocionais e funcionais provocados, visando à universalização de critérios e às comparações entre populações afetadas ${ }^{13,16}$.

Em estudos realizados com população de idosos, questionários foram aplicados englobando as características do zumbido e a repercussão na vida do paciente ${ }^{3,13,14}$. Nessa população, este é tido como causa da diminuição da inteligibilidade da fala, dificultando seu relacionamento interpessoal. A maioria dos pacientes referiu ainda interferência no sono e no estado emocional, com implicação também na realização das atividades de vida diárias ${ }^{13,14}$

É descrito na literatura que o zumbido é potencializado quando ocorre juntamente com algum tipo de perda auditiva ${ }^{3,9-12,14}$. Foi realizada pesquisa com indivíduos brasileiros de 7 a 95 anos, todos com queixa de zumbido. Concluiu-se que a ocorrência de perda auditiva em sujeitos portadores de zumbido é progressivamente maior à medida que a idade avança, com maior incidência também de perdas auditivas ${ }^{10,14,15}$.

No teste Audiometria Tonal, mensura-se a intensidade mínima audível para puros. A configuração da curva audiométrica de um paciente que possui zumbido é geralmente descendente com audição pior nas frequências altas $3,6,11,17,18$.

Em estudo realizado em população com zumbido, objetivou-se comparar as características clínicas e sua interferência nas atividades da vida diária em pacientes com e sem perda auditiva. Concluiu-se que pacientes sem perda auditiva associada apresentaram menor interferência nas atividades de concentração e equilíbrio emocional, quando comparados com o grupo controle, que possuía perda auditiva ${ }^{16}$.

A Fonoaudiologia insere-se tanto na avaliação audiológica do paciente quanto na terapia para o zumbido. São descritas na literatura a utilização de aparelhos de amplificação sonora individual (AASI) e até mesmo implante colear, como controle do zumbido em pacientes com perda auditiva neurossensorial ${ }^{19}$. A reabilitação vestibular também foi descrita como opção na melhoria da sintomatologia no caso de paciente com zumbido associado a alterações vestibulares, com remissão ou diminuição significante do impacto do zumbido após a terapia ${ }^{20-22}$. Além desses é famosa a opção pela terapia de habituação, o TRT - Tinnitus Retraining Therapy, aplicado por fonoaudiólogo devidamente treinado ${ }^{23}$.

Diante da grande subjetividade e das controvérsias em torno deste tema, a presente pesquisa visa analisar a influência do zumbido na qualidade de vida de seus portadores, bem como correlacionar o perfil audiométrico, verificando a existência de relação entre grau de incômodo e presença de perda auditiva concomitante, além de relação dos fatores sexo e idade. Espera-se que tais dados auxiliem no direcionamento da terapia e, consequentemente, na melhoria da qualidade de vida desse sujeito. 


\section{MÉTODO}

O delineamento do estudo é do tipo observacional, transversal, com amostra de conveniência. Realizou-se amostragem piloto, com casuística composta por 22 participantes, encaminhados ao Serviço de Audiologia do Hospital São Geraldo, anexo do Hospital das Clínicas-UFMG em Belo Horizonte, por otorrinolaringologistas e/ou neurologistas, para realização do exame Potenciais Evocados Auditivos de Tronco Encefálico (PEATE), que apresentavam como queixa a presença de zumbido. Foram incluídos na pesquisa participantes com idade acima de 18 anos, de ambos os gêneros, que já haviam realizado a Audiometria Tonal e traziam consigo os resultados apresentados no momento da execução do exame PEATE. Foram excluídos da amostra pacientes com quaisquer comprometimentos cognitivos, neurológicos, ou não usuários do código linguístico oral. Todos os participantes receberam as orientações e explicações sobre a pesquisa, leram, concordaram e assinaram o Termo de Consentimento Livre e Esclarecido.

Esse estudo foi aprovado pelo Comitê de Ética em Pesquisa da Universidade Federal de Minas Gerais, em agosto de 2009 com o parecer no 295/09.

Inicialmente, foi aplicada anamnese prevista no protocolo de atendimento do Ambulatório de Audiologia, enfocando história atual e pregressa da queixa apresentada pelo paciente, bem como sinais e sintomas associados. Em seguida, aplicado questionário enfocando descrição das características clínicas do zumbido, repercussão do zumbido na vida do paciente ${ }^{11}$.

A severidade do zumbido foi analisada pela escala análoga visual (EVA). Segundo esse método, o paciente é inquirido a dar nota de 1 a 10 a seu zumbido, imaginando que 1 seria zumbido leve, e 10 o pior zumbido que ele poderia imaginar (figura 1). Para realização de análise estatística, fragmentaram-se os resultados da EVA em maiores (muito incômodo) ou menores (pouco incômodo) que cinco. Foi realizada adicionalmente coleta dos dados da Audiometria Tonal Limiar, Vocal e Imitanciometria. A classificação do grau de perda auditiva foi baseada em critérios propostos por Davis \& Silverman ${ }^{24}$.

Adotou-se o software Statistical Package for Social Sciences (SPSS) 15.0 for windows - SPSS Incorporation, Chicago, Illinois, Estados Unidos da América, 2008.

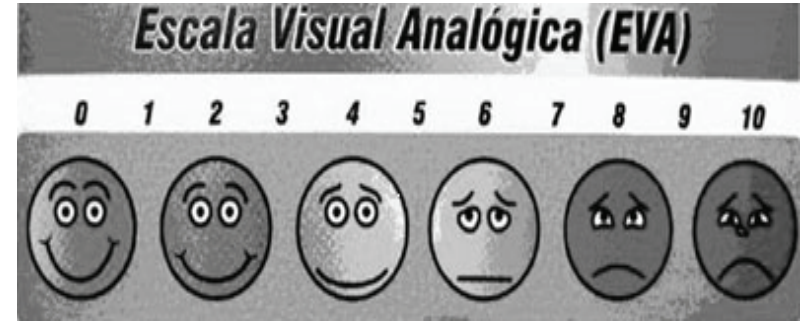

\section{Figura 1 - Modelo de Escala Visual-Analógica (EVA)}

Para caracterização da amostra, foram feitas tabelas de distribuição de frequências para as variáveis categóricas (sexo, presença de perda auditiva, interferência no sono, na concentração, na emoção e na vida social, tipo de perda auditiva) e, para as variáveis contínuas (idade, resultado do EVAS), foram utilizadas

medidas de tendência central (média, mediana, mínimo e máximo) e variabilidade (desvio padrão (DP)).

Para comparação dos grupos com variáveis categóricas, foi feito o teste Qui-quadrado de Pearson, apropriado para comparação de proporções, ou teste de Fisher, quando foram utilizadas amostras com pequenas frequências.

Com a finalidade de estudar a relação estatística entre as entre resultado do EVAS e idade do pacientes, utilizou-se a regressão. Os resultados foram demonstrados por meio do diagrama de espalhamento ou de dispersão (Scatter plot).

Em todas as análises, foi considerado um nível de $5 \%$ de significância.

\section{RESULTADOS}

O grupo estudado foi composto por 22 sujeitos de ambos os sexos. Foram avaliados 11 pacientes do sexo feminino e 11 do sexo masculino. A média de idade da população estudada foi de 53,09 anos, com desvio padrão igual a 13,71, mínimo 22 e máximo 73.

Quanto ao perfil audiométrico, dos 22 pacientes 7 $(31,8 \%)$ apresentaram audição normal e 15 (68,2\%) apresentaram algum tipo de perda auditiva. Não ocorreu na amostra caso algum de paciente com comprometimento retrococlear A tabela 1 descreve o perfil audiométrico dos pacientes em relação ao tipo e grau de perda auditiva. Todos os pacientes da amostra possuem zumbido. 
Tabela 1 - Perfil audiométrico dos pacientes em relação ao tipo e grau de perda auditiva ( $n=15)$

\begin{tabular}{ccccc}
\hline \multirow{2}{*}{ Tipo de perda auditiva } & \multicolumn{2}{c}{ Orelha direita } & \multicolumn{2}{c}{ Orelha esquerda } \\
\cline { 2 - 5 } & $\mathbf{N}$ & $\%$ & $\mathbf{N}$ & $\%$ \\
\hline PANS LEVE & 2 & 9,1 & 3 & 13,6 \\
PANS MODERADA & 4 & 18,2 & 4 & 18,2 \\
PAM LEVE & 0 & 0 & 1 & 4,5 \\
PAM MODERADA & 0 & 0 & 4 & 18,2 \\
\hline
\end{tabular}

Legenda:

$\mathrm{N}$ - número de sujeitos

PANS - Perda auditiva neurossensorial leve

PAM- Perda auditiva mista

Na tabela 2, é descrita a associação entre perda auditiva e o resultado da EVA.

Tabela 2 - Associação entre presença de perda auditiva e resultado do EVAS $(\mathrm{n}=22)$

\begin{tabular}{cccc}
\hline \multirow{2}{*}{ EVAS } & \multicolumn{2}{c}{ Perda Auditiva } & P \\
\cline { 2 - 3 } & Sim & Não & \\
\hline $\mathbf{5} 5$ & 4 & 1 & $\mathbf{0 , 4 7 7}$ \\
\hline $\mathbf{5}$ & 11 & 6 & \\
\hline
\end{tabular}

A figura 2 demonstra os resultados da influência do zumbido na qualidade de vida de seus portadores.

O estudo de correlação entre intensidade do zumbido (EVAS < 5 e EVAS >5) e influência na qualidade de vida está demonstrado na tabela 3.

A figura 3 demonstra que não há correlação entre idade e resultado de EVAS ( $p>0,05)$.

A análise comparativa em relação ao gênero não demonstrou diferença com significância estatística, resultando em $\mathrm{P}$ valor maior que 0,005.

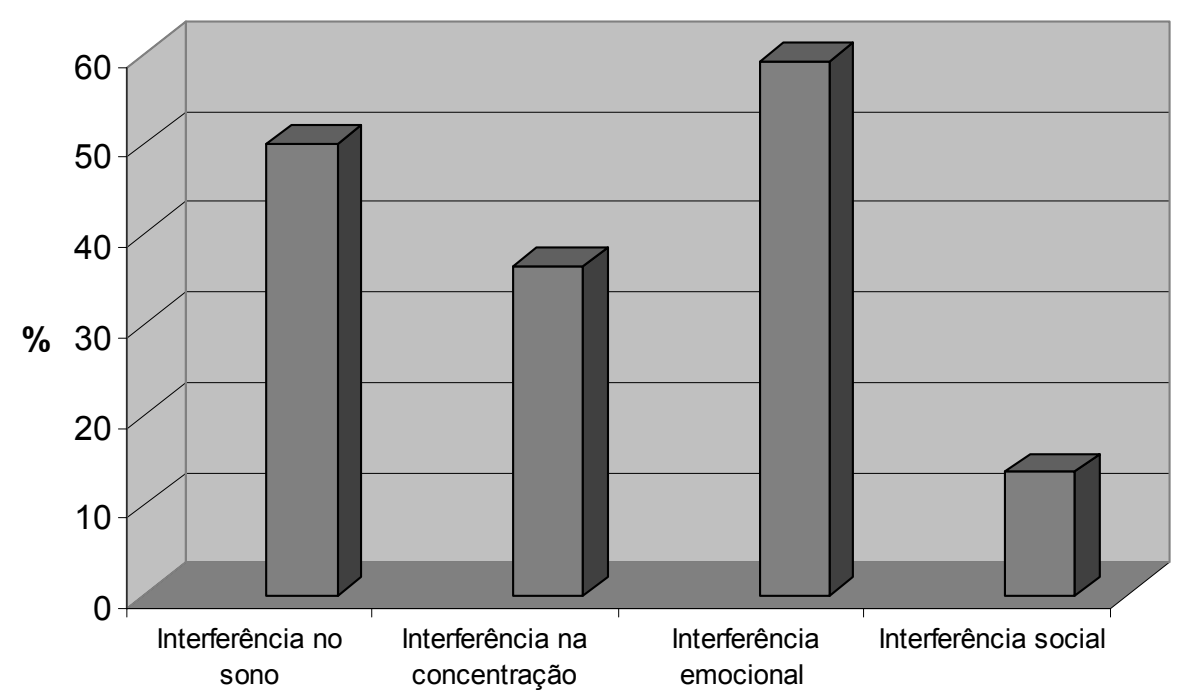

Figura 2 - Influência do zumbido na qualidade de vida de seus portadores $(n=22)$

Tabela 3 - Correlação entre intensidade do zumbido (EVAS < e EVAS>5) e influência na qualidade de vida

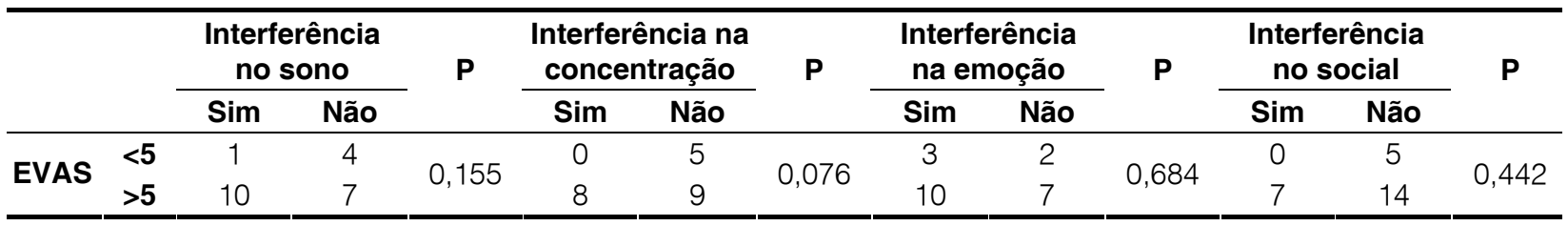

$\mathbf{P}=$ probabilidade de significância: Teste exato de fisher. 


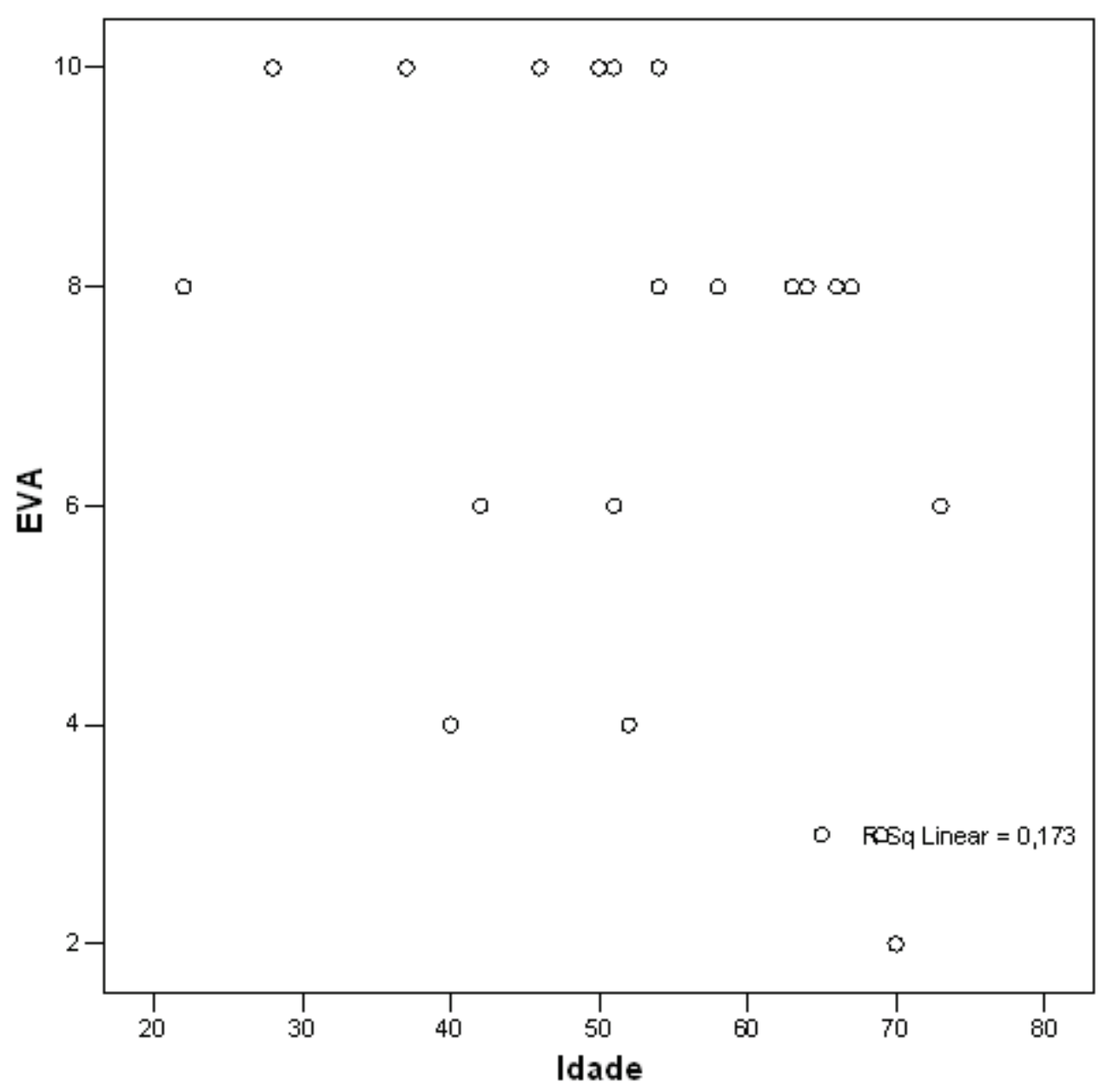

Figura 3 - Diagrama de espalhamento referente à idade e EVA

\section{DISCUSSÃO}

Ao analisar a idade da população estudada, nota-se média de 53,09 anos. Apesar de possuir alto desvio padrão, este dado está de acordo com a literatura, que descreve que a ocorrência de zumbido é maior em indivíduos a partir de 50 anos, apesar da variabilidade de idade de incidência ${ }^{3,8,11,12,16,17}$.

Quanto ao perfil audiométrico da população, observa-se que $68,2 \%$ dos sujeitos estudados $(n=15)$ apresentaram perda auditiva concomitante ao zumbido (Tabela 1).

Dos pacientes possuidores de perda auditiva, 12 apresentaram perda auditiva neurossensorial em pelo menos uma das orelhas, e três perda mista uni ou bilateral.

É descrito na literatura que $80 \%$ dos zumbidos são de origem neurossensorial, ou seja, proveniente da contração das células ciliadas externas. Este estudo está de acordo com a literatura, uma vez que $58,1 \%$ dos sujeitos possuíram componentes neurossensoriais em alterações auditivas ${ }^{9,10,12}$.
É incerto se a perda auditiva funcionaria como gatilho para o início do zumbido ou se seria também preditor de sua gravidade. No caso de pacientes com perda auditiva e zumbido concomitantemente, sugere-se investigar qual desses sintomas provoca maior incômodo, a fim de indicar qual melhor abordagem deve ser realizada durante a terapia ${ }^{3,10,14,15}$. Pode-se observar que $50 \%(n=11)$ dos pacientes que possuiam perda auditiva relataram maior incômodo referente ao zumbido (Tab 2). No entanto, 4 pacientes $(18,18 \%)$ possuidores de perda auditiva relatam menor incômodo. $27,27 \%$ dos pacientes com audição normal apresentaram zumbido com alto grau de incômodo.

Ofato de alguns pacientes apresentarem zumbido com audição normal e, ainda, apresentarem grau de incômodo pode ser explicado por fatores associado à queixa de zumbido. Adversidades, como fadiga, estresse, ansiedade e depressão levam a uma piora no grau de incômodo do zumbido percebido pelo paciente, o que demonstra que não é necessária perda auditiva concomitante para que o grau de incômodo seja relatado como moderado 
ou severo. Queixas, como: alteração vestibular, dor de cabeça e infecção das vias aéreas superiores, quando concomitante ao zumbido, também agravam o grau de incômodo descrito por pacientes ${ }^{16}$. $\mathrm{Na}$ amostra estudada, verificou-se que pacientes sem perda auditiva apresentaram menor grau de incômodo quando comparado aos pacientes com perda auditiva. No entanto, não foi questionado a esses pacientes se possuíam outras alterações concomitantes ao zumbido. Assim, não se pode afirmar que a ausência de perda auditiva está diretamente relacionada com a redução do incômodo. Os achados corroboram, ainda, o modelo proposto por Jastreboff 7,8 , demonstrando que a influência do Sistema Límbico e as emoções podem agir mais negativamente sobre o incômodo gerado pelo zumbido do que o grau da perda auditiva.

Em estudo realizado na Austrália, foi observado que $20 \%$ da população apresentava zumbido com graus intensos de incômodo ${ }^{9}$.No presente estudo observou-se que $77,28 \%$ da amostra $(n=17)$ relataram muito incômodo utilizando-se a escala análogo-visual (Tab 2). Apenas cinco pacientes $(22,72 \%)$ consideraram o zumbido como sintoma que não gerava grande incômodo Tal incidência é acima do esperado. Esse achado é clinicamente importante. No entanto, o número total de sujeitos avaliados nesta pesquisa não é expressivo, quando comparado à incidência na população afetada por tal sintoma, não se podendo, assim, generalizar tal dado.

A análise de regressão confirma a ausência de correlação entre as variáveis idade e EVA $(R 2=0,173)$. Não foi possível observar no perfil da população associação entre o aumento da idade e aumento do incômodo impactado pelo zumbido, uma vez que os resultados encontram-se dispersos e sem padrão nem organização. Esse achado difere da literatura, que demonstra o aumento do incômodo gerado pelo zumbido associado ao aumento de idade de seus portadores. 3,9-12,14.

Em relação ao impacto na qualidade de vida, analisaram-se separadamente as variáveis: interferência no sono, interferência na concentração, interferência no estado emocional e na vida social. Esses dados estão distribuídos na Figura 2. É possível observar que $59,09 \%(n=13)$ dos pacientes consideraram que o zumbido interferia em seu estado emocional; $50 \%$ dos pacientes relataram impacto na qualidade do sono; $36,36 \%(n=8)$ impacto na taxa de concentração e $31,81 \%(n=7)$ interferência em sua vida social.

Em estudo realizado em população paulista, encontrou-se a incidência da interferência no sono em $50 \%$ da população estudada ${ }^{6}$, similar ao encontrado no presente estudo. No entanto, ao comparar os achados do presente estudo com a literatura, nota-se diferença marcante do tamanho na população estudada, sendo o " $n$ " deste estudo quantitativamente menor.

Quanto à interferência na concentração, 35 \% da população relatou ter alterações de concentração geradas pelo zumbido e 15 sujeitos (65\%) não relataram alteração na concentração (Tab 3). Tal resultado foi inferior ao obtido por Sanchez (2004), no qual a interferência na concentração foi queixa de $43,5 \%$ (8 sujeitos) da população. Vale ressaltar que $0 n$ das duas pesquisas diferem, o que pode ter sido uma possível causa de resultados distintos encontrados.

Ao analisar a interferência do zumbido no estado emocional e sua relação com o grau de incômodo relatado pelo paciente, 8 pacientes $(36,36 \%)$ relataram interferência de alguma forma em suas emoções. Estes resultados aproximam-se muito dos resultados encontrados por Sanchez (2004) onde $59 \%$ da população estudada relatou interferência no equilíbrio emocional.

No presente estudo não houve associação entre o grau de incômodo do zumbido e a interferência na vida social. Esses dados corroboram estudos prévios ${ }^{6}$.

Pacientes com queixa de zumbido podem apresentar graus de incômodos variados, tendo maior ou menor impacto na qualidade de vida. Tendo em vista a definição de qualidade de vida proposta pela OMS em $1995^{24}$, sabe-se que a percepção subjetiva que o paciente tem sobre seu bem-estar é relevante para contextualizar o grau de impacto do sintoma relatado em sua vida. Quando se aplica esse conceito ao paciente que possui zumbido não se pode perder de vista que queixas são relatadas, e se há algum grau de fobia relacionado aos danos. É relevante lembrar que o zumbido pode afetar atividades da vida diária, bem como atividades mentais superiores, levando ao isolamento social e até mesmo ao suicídio. Sendo assim, é importante entender detalhadamente como o zumbido impacta a qualidade de vida do paciente, sugerindo dados que facilitarão a conduta terapêutica.

Neste estudo, foi evidenciado que o zumbido impacta mais as emoções e o sono dos pacientes do que a concentração e realização de atividades sociais.

Apesar de relevantes, as conclusões dessa pesquisa não podem ser extrapoladas para outras populações. Supõe-se que a ausência de associação significativa e/ou correlação nas análises realizadas não tenha ocorrido por se tratar de um estudo piloto, com $n$ insuficiente para estabelecer tais tipos de relação e associação estatística. 


\section{CONCLUSÕES}

Dos 22 pacientes com zumbido encaminhados para realização do PEATE, $68,19 \%$ apresentavam algum tipo de perda auditiva uni ou bilateral. Não foi encontrada relação estatisticamente significante entre a presença de perda auditiva e grau de incômodo relatado. $27,27 \%$ dos pacientes com audição normal apresentaram zumbido com alto grau de incômodo. No entanto, não houve relação entre a presença de zumbido e perda auditiva associada. Não houve ainda relação entre o grau de incômodo e presença de perda.

\begin{abstract}
Purpose: to analyze life quality in patients that suffer from tinnitus; to check the relation between degree of tinnitus' annoyance and presence of concomitant hearing loss. Method: transversal observational study, , with convenience sample. Anamneses were applied focusing tinnitus symptom over 22 patients. In order to analyze data we used Fisher's Exact Test within a 5\% significance level. Results: $31.8 \%$ of the sample had tinnitus concomitant with normal hearing bilaterally; $68.2 \%$ had tinnitus concomitant with some type of hearing loss in one or both ears. In this case, 12 patients showed sensorioneural hearing loss and 03 patients had mixed loss. As for the degree of annoyance referred from tinnitus symptom, using the visual analog scale, 17 (77.27\%) patients reported intense discomfort, whereas $5(22.72 \%)$ reported restricted discomfort. Regarding tinnitus' interference in the quality of life, it was possible to note that tinnitus impacts most in patients' emotions and sleep. Conclusions: from 22 patients referred to take PEATE's exam, $70 \%$ of the survey had hearing loss concomitant with tinnitus. However, the relation between tinnitus and hearing loss was not evidenced. We neither found any relation between degree of hearing loss and annoyance.
\end{abstract}

KEYWORDS: Tinnitus; Hearing Loss; Quality of Life; Audiology

\section{REFERÊNCIAS}

1. National Institutes of Health, Consensus Development Program Statements, 1996. Disponível em: http://consensus.nih.gov/HISTORICALSTATE MENTS.htm

2. Gopinath B, McMahon CM, Rochtchina E, Karpa MJ, Mitchell P. Incidence, Peristence and Progression of Tinnitus Symptons in Older Adults: The Blue Mountains Hearing Study. Ear \& Hearing. 2010;31(3):407-12.

3. Pinto PC, Sanchez TG, Tomita S. Avaliação da relação entre severidade do zumbido e perda auditiva, sexo e idade do paciente. Braz J Otorhinolaryngol. 2010;76(1):18-24.

4. Gomes SJ, Barboza MS, Santos TMM. A incidência de zumbido numa amostra aleatória na cidade de Salvador. CEFAC,2004;(6)89-93.

5. Mor R, Azevedo MF. Emissões otoacústicas e sistema olivococlear medial: pacientes com zumbido sem perda auditiva. Pró-fono,2005;(17):283-92.

6. Sanchez TG, Ferrari GMS. O que é zumbido? In: Samelli AG. Zumbido, avaliação,diagnóstico e reabilitação. São Paulo : Lovise 2004. p17-22.
7. Jastreboff PJ. Phantom auditory perception (Tinnitus). Mechanism of generation and perception. Neurosci Res. 1990; (8):221-54.

8. Jastreboff PJ, Hazzel J. A neurophysiological approach to tinnitus: clinical implications. British Journal of Audiology.1993; (27):7-17.

9. Sindhusake D, Golding M, Wigney D, Newall P, Jakobsen K, Mitchell P. Factors Predicting Severity of Tinnitus : A population-Based Assessment. J Am Audiol. 2004; (15): 269-80.

10. Gonçalves MS, Tochetto TM, Rossi AG. Condição auditiva de indivíduos com queixa de zumbido. Saúde.2005;(31):5-9.

11. Sanches TG, Medeiros IRT, Levy CPD, Ramalho JRO, Bento RF. Zumbido em pacientes com audiometria normal : caracterização clínica e repercussões. Rev Bras Otorrinolaringol 2005; 4: 427-31.

12. Person OC, Feres MCLC, Barcelos CEM, Mendonça RR, Marone MR, Rapoport PB. Zumbido: aspectos etiologicos, fisiopatologicos e descricao de um protocolo de investigação. Arq Med ABC. 2005;30(2):111-8.

13. Teixeira AR, Nunes MGP, Freitas CR, Gonçalves AK, Teixeira SB. Análise da Qualidade 
de Vida de Idosos com Sintoma de Zumbido. Arq. Int. Otorrinolaringol., Jan/Fev/Março - 2010; 14(1):54-9.

14. Ferreira LMBM, Ramos Jr NA, Mendes EP. Caracterização do zumbido em idosos e de possíveis transtornos relacionados. Braz J Otorhinolaryngol. 2009; 75(2): 249-55

15. Dias A, Cordeiro R. Interação entre grau de perda auditiva e o incômodo com zumbidos em trabalhadores com história de exposição ao ruído. Ver Bras Otorrinolaringol 2008; 74(6): 876-83.

16. Azevedo AA, Oliveira PM, Siqueira AG, Figueiredo RR. Análise crítica dos métodos de mensuração do zumbido. Rev Bras Otorrinolaringol. 2007;73(3):418-23.

17. Figueiredo RR, Azevedo AA, Oliveira PM. Análise da correlação entre a escala visuo-análoga e o Tinnitus Handicap Inventory na avaliação de pacientes com zumbido. Rev Bras Otorrinolaringol 2009; 75(1):76-9.

18. Dias A, Cordeiro R, Corrente JE, Gonçalves CGO. Associação entre perda auditiva induzida pelo ruído e zumbidos. Cad Saúd. Pública. 2006;22(1):63-8.
19. Moura LOS, lório MCM, Azevedo MF. A eficácia da adaptação de prótese auditiva na redução ou eliminação do zumbido. Rev Bras Otorrinolaringol.2004;(70):524-31.

20. Holdefer L, Oliveira CACP, Venosa AR. Sucesso no tratamento do zumbido com terapia em grupo. Braz J Otorhinolaryngol. 2010;76(1):102-6.

21. Zeigelboim BS, Rosa MRD, Klagenberg KF, Jurkiewicz AL. Reabilitação vestibular no tratamento da tontura e do zumbido. Rev Soc Bras Fonoaudiol. 2008;13(3):226-32.

22. Knobel KAB, Pfeilsticker LN, Stoler G, Sanchez TG. Contribuição da reabilitação vestibular na melhora do zumbido: um resultado inesperado. Rev Bras Otorrinolaringol. set./out. 2003; 69(6) :779-84. 23. Jastreboff $P$, Hazell J. TRT - Tinnitus Retraining Therapy.. Disponível em: http://www.tinnitus.org/ 24. Davis H, Silverman SR. Auditory Test Hearing Aids. In: DAVIS H, SILVERMAN SR Hearing and Deafness, Holt: Rinehart and Winston, 1970.

25. Fleck MP, Chachamovich E, Trentini C. Development and validation of the Portuguese version of the WHOQOL-OLD module. Rev Saúde Pública. 2006;(40):5.
http://dx.doi.org/10.1590/S1516-18462011005000107

RECEBIDO EM: 21/02/2011

ACEITO EM: 11/05/2011

Endereço para correspondência:

Carolina Campos Esteves

R. Raimundo Correa, 108/100, São Pedro

Belo Horizonte - MG - Brasil

CEP: 30330-090

E-mail: carolcampose@ hotmail.com 